\title{
Physicochemical characterisation of degrading polycaprolactone scaffolds
}

\author{
Lucy A. Bosworth*, Sandra Downes \\ The University of Manchester, School of Materials, Grosvenor Street, Manchester, Lancashire M1 7HS, United Kingdom
}

\section{A R T I C L E I N F O}

\section{Article history:}

Received 26 October 2009

Received in revised form

18 August 2010

Accepted 22 September 2010

Available online 1 October 2010

\section{Keywords:}

Hydrolytic degradation

Polycaprolactone

Electrospinning

Surface area

Crystallinity

\begin{abstract}
A B S T R A C T
A degradation study investigating the hydrolysis of different scaffolds of polycaprolactone suspended in phosphate buffer solution at $37^{\circ} \mathrm{C}$ was performed over a three month period. Structures included electrospun fibres, held as 2D mats and 3D bundles, and solvent cast films. These structures and their surrounding solutions were physiochemically characterised using a range of techniques. Changes in scaffold physicochemical properties were observed over the course of the study, including significant loss in molecular mass, increases in thermal properties and crystallinity, and increases in tensile properties. The presence of degradation products, such as capronic acid containing compounds was also identified in the surrounding solution. 3D electrospun bundles - as a consequence of being the least crystalline scaffold and hence most susceptible to hydrolysis - demonstrated greatest reduction in molecular mass over the three months, followed by 2D electrospun mats, and the lowest mass loss was observed for solvent cast films.
\end{abstract}

(c) 2010 Elsevier Ltd. All rights reserved.

\section{Introduction}

Scaffolds engineered to stimulate tissue regeneration in vivo, must be able to provide necessary mechanical support until sufficient ingrowth from the body's natural tissue has occurred. Once this has happened the new tissue should be able to withstand various stresses that may be placed on it [1]. Ideally, the scaffold should be degradable; eliminating the need for surgical removal, with its rate of degradation matching the rate of new tissue formation. If the rates are not synchronised the neo-tissue may not be sufficiently stable and could compromise the implant's success.

The terminology used to define synthetic polymer breakdown is not consistent through the literature. "Biodegradable" is most commonly used, although this is misleading upon close examination of the breakdown of the polymer in question. In order to accurately describe the breakdown mechanism, Vert [2] proposed the following terminologies for clarification - biodegradable, bioresorbable, bioabsorbable and bioerodible (Table 1 ).

PCL is commonly referred to as a "biodegradable" polyester $[3,4]$ and whilst this material is known to breakdown in vivo, the breakdown products form naturally occurring metabolites, which are readily metabolised by the body and eliminated without toxicity. In this respect, the correct terminology for PCL is "bioresorbable" as used by Lam [5]. However, in certain circumstances the term "bioerodible" may also be applied; as discovered by Pitt

\footnotetext{
* Corresponding author.

E-mail address: lucy.bosworth@manchester.ac.uk (L.A. Bosworth).
}

[6], who investigated the enzymatic surface erosion of PCL both in vitro and in vivo.

PCL can undergo two types of degradation - hydrolytic and enzymatic - which both yield carboxyl-group containing oligomers. Degradation via hydrolysis occurs mainly in the bulk of the material, whereas enzymatic degradation favours surface erosion.

\subsection{Hydrolysis of $P C L$}

PCL degrades, in the first instance, by random chain scission of the ester groups in the material bulk via hydrolysis [7]. This preferentially occurs in the polymers amorphous regions due to loose structural packing within this area of the polymer, making ester bonds more exposed to attack from water molecules [8,9]. Ester bonds are subsequently cleaved from the polymer backbone shortening the chain lengths [10] resulting in the formation of a carboxylic acid known as capronic acid (synonyms; 6-hydroxycaproic acid/6-hydroxyhexanoic acid) (Fig. 1). Rearrangement of cleaved chains ensues, which can lead to ordered packing that maintains or increases the level of crystallinity [7,9]. Local generation of carboxylic acid by-products, if not removed, can increase the surrounding acidity promoting autocatalysis, which subsequently accelerates the rate of degradation [11].

Polymers capable of undergoing hydrolytic degradation initially absorb water into the material bulk, hydrating the molecular chains. The type of functional group present along the length of the polymer chain affects the rate of water absorption, its subsequent hydrolysis and ultimately rate of degradation [12]. Thus the 
Table 1

Clarification of terminology and their definitions with respect to the breakdown of synthetic polymers [2].

\begin{tabular}{ll}
\hline Terminology & Definition \\
\hline Biodegradable & $\begin{array}{l}\text { For polymeric systems that undergo macromolecular } \\
\text { breakdown with dispersion in vivo, but without proof } \\
\text { of its elimination. (Excludes biodegradation by } \\
\text { environmental, fungi or bacterial means). } \\
\text { For polymeric systems which degrade whilst in vivo } \\
\text { and are further resorbed by natural metabolism for } \\
\text { total elimination. }\end{array}$ \\
Bioabsorbable & $\begin{array}{l}\text { For polymeric systems that dissolve in the presence } \\
\text { of bodily fluids without chain cleavage and changes in } \\
\text { molecular mass. }\end{array}$ \\
& $\begin{array}{l}\text { For polymeric systems which experience degradation } \\
\text { on its material surface. }\end{array}$ \\
\hline
\end{tabular}

hydrophobic nature of the PCL backbone should limit the rate of water absorption, slowing the degradation rate.

The degradation rate of PCL varies depending on its structural and morphological formations and surface area to volume ratio $[13,14]$. Bölgen [10] proposed that samples with a higher surface area to volume ratio degraded faster due to greater penetration of water into the sample, initiating increased rates of chain scission. Conversely, Athanasiou [15] investigated percentage porosity within PLGA scaffolds and proved those with higher porosity degraded at a slower rate compared to those of $0 \%$ porosity; reasoning that a greater surface area allowed easier removal of acidic breakdown products, thus reducing the likelihood of autocatalysis.

\subsection{Enzymatic degradation of $P C L$}

Enzymatic degradation occurs at the material surface, where hydrolytic enzymes adsorb onto the surface before initiating polymer hydrolysis [16]. PCL is susceptible to attack from an esterlike lipase enzyme, with cleavage occurring at the ester bond due to its structural similarity to lipids (i.e. carboxylic acid end group of a fatty acid is bonded to an alcohol containing compound, such as glycerol, creating an ester bond) [17].

For glassy materials, the crystallinity holding polymer chains in place inhibits enzyme activity, and is only then instigated after the initial release of lower molecular weight by-products [6]. For rubbery materials, the mobility of polymer chains increases their exposure to enzymes and hence can significantly affect their rates of degradation [6].

Electrospinning creates fibre matrices of high surface area to volume ratio and consequently provides large areas of contact to instigate rapid degradation by enzyme activity; as demonstrated by Ito [18], whereby electrospun poly(3-hydroxybutyrate-co-3hydroxyvalerate) (PHBV) fibres degraded at a faster rate than PHBV films. A similar rate of degradation was also observed by Zeng [16] for

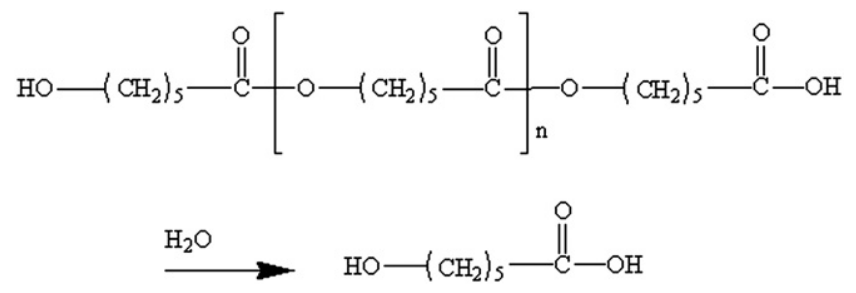

Fig. 1. Effects of hydrolytic degradation on PCL to form capronic acid (6-hydroxycaproic acid) [23]. electrospun polycaprolactone-triethylbenzylammonium chloride (PCL-TEBAC) fibres, where degradation in the presence of the enzyme, lipase, at $37^{\circ} \mathrm{C}$, resulted in rapid bioerosion of the polymer fibres after a short period of $8.5 \mathrm{~h}$.

\subsection{Rate of PCL degradation}

The rate of PCL degradation is dependent on its structural formation. Bölgen [10] demonstrated that whilst the surface area to volume ratio of a material affects the rate of degradation, its physical shape is also important. Their in vitro assessment of PCL electrospun fibres and solvent cast films demonstrated a slower degradation rate for the fibres. Explanations included the effect of electrospinning on wettability and the diffusion of breakdown products. PCL fibres were more hydrophobic compared to PCL films, with lower adhesion of water molecules to the fibres surface, subsequently reducing surface hydrolysis and the diffusion of water molecules into the fibrous structure [10]. For electrospun fibres, the diffusion of breakdown products out of the material bulk will be of shorter distance due to the fibres large surface area to volume ratio, enabling easier removal and reducing the effects of autocatalysis compared to materials of smaller surface area to volume ratio [13]. The in vitro study by Bölgen [10] was performed in Ringer's solution; if, however, the study was performed in an enzyme-containing solution the degradation profile may have been completely transformed. This was demonstrated by Gan [19], who submerged PCL solvent cast films in PBS containing lipase at $37^{\circ} \mathrm{C}$; complete degradation occurred after four days.

As a material regularly incorporated in the field of biomaterials, this paper details PCL structures with varying conformations, as a consequence of different processing methods - electrospinning and solvent casting - which were investigated in terms of their hydrolytic degradation behaviour over a three month period. It was hypothesised that these different PCL structures will undergo differing levels of hydrolysis as a consequence of surface area to volume ratio. It was surmised that structures with largest surface area would experience greatest degradation over the 90 day investigation. This study aims to determine the effects of surface area to volume ratio on PCL structures in terms of their bulk physicochemical degradation properties.

\section{Materials and methods}

\subsection{Preparation of PCL samples}

All PCL samples, electrospun 2D aligned-fibrous mats, 3D electrospun fibrous bundles and solvent cast films, were fabricated from a single solution of PCL $\left(\mathrm{M}_{n} 80,000 \mathrm{gmol}^{-1}\right.$, Sigma Aldrich) dissolved in Acetone (analytical grade, Fisher Scientific) at a concentration of $10 \% \mathrm{w} / \mathrm{v}$.

\subsubsection{Electrospun PCL fibres}

The electrospinning parameters employed for the fabrication of the $2 \mathrm{D}$ and 3D fibre matrices were as follows: voltage $20 \mathrm{kV}$ (Series 120 Watt regulated high voltage DC power supply, Glassman High Voltage, Inc), flow-rate $0.05 \mathrm{ml} \mathrm{min}^{-1}$ (SP230IW2, World Precision Instruments), needle-tip ( $\varnothing 0.8 \mathrm{~mm}, \mathrm{BD}$ Microlance) to collector distance $20 \mathrm{~cm}$ and spinning time for each fibre collection $15 \mathrm{~min}$. A purpose-made aluminium mandrel $(\varnothing 120 \mathrm{~mm}$, edge thickness $3 \mathrm{~mm}$ ) rotating at 500RPM was used to collect the fabricated fibres. Collected fibres were removed from the mandrel as a single 2D strip, which was then cut every $3 \mathrm{~cm}$ along its length. Half of these $3 \mathrm{~cm} 2 \mathrm{D}$ electrospun mats were manually twisted to create $3 \mathrm{D}$ 
fibrous bundles. Twisting was achieved by holding either end of the mat and twisting along its length.

\subsubsection{Solvent cast PCL films}

Solvent cast films were formed by evenly smoothing $1 \mathrm{ml} \mathrm{PCL} /$ Acetone solution over the surface of a dust-free glass slide $(76 \times 26 \mathrm{~mm})$ (Thermo Scientific). Once dry, films were cut to the same dimensions of the $2 \mathrm{D}$ fibrous mats $(3 \times 30 \mathrm{~mm})$.

\subsection{Degradation set-up}

All samples were fabricated on the same day and individually suspended in eppendorfs (Fisherbrand) containing $1.5 \mathrm{ml}$ sterile Phosphate Buffer Solution (PBS) (Invitrogen). All eppendorfs were covered in lab-film and housed within a $37^{\circ} \mathrm{C}$ oven (Binder). The degradation study was performed for 90 days; at each time-point, samples were taken from the oven, the PBS was removed, samples were blotted with towelling and then left to dry under atmospheric conditions (approximately $1 \mathrm{~h}$ ) prior to testing. The exposed surface area of the fibres and films that is in direct contact with the PBS is as follows; electrospun 2D fibrous mat $-190.56 \mathrm{~mm}^{2}$, electrospun 3D fibrous bundle $-33.18 \mathrm{~mm}^{2}$ and solvent cast film $193.86 \mathrm{~mm}^{2}$ (Table 2).

\subsection{Characterisation methods}

\subsubsection{Gel permeation chromatography (GPC)}

The molecular weight distributions of the samples were determined using GPC (Applied Chromatography Systems, Ltd). Samples were individually dissolved in tetrahydrofuran (THF) (Fisher Scientific) (concentration $0.2 \% \mathrm{w} / \mathrm{v}$ ). $100 \mu$ l of solution was injected into the GPC, which had been previously calibrated with polystyrene (PS) standards (Pressure chemical standards) in THF with known molecular weights ranging from $600-7.7 \times 10^{6} \mathrm{~g} \mathrm{~mol}^{-1}$. Distilled THF with flow rate $1 \mathrm{ml} \mathrm{min}^{-1}$ was used as the mobile phase. The column (Phenomenex) was composed of Phenogel $5 \mu \mathrm{m}$, with pore sizes $500 \AA, 5 \times 10^{4} \AA$ and $5 \times 10^{6} \AA$. For each scaffold type, three samples were collected, each of which was analysed in triplicate. The average molecular mass distributions were determined using PSS Win GPC software.

\subsubsection{Differential scanning calorimetry (DSC)}

DSC analysis was performed using DSC Q100 (TA Instruments) with samples exposed to a temperature range from -100 to $100{ }^{\circ} \mathrm{C}$ and a heating rate of $10^{\circ} \mathrm{C} \mathrm{min}-1$. Data from this thermal heat was analysed as the thermal history of the material, at this point, had not been removed and any effects from the material processing, i.e. solvent casting and electrospinning techniques, could be identified. Universal Analysis 2000.v.4.2E software (TA Instruments) was used to quantify the melting temperature $\left(T_{\mathrm{m}}\right)$ and enthalpy of fusion $\left(\Delta H_{\mathrm{m}}\right)$ for each sample type. The change in material crystallinity was determined by comparing the enthalpy of fusion with that for $100 \%$ crystalline PCL (135.44 $\mathrm{Jg}^{-1}$ ) as measured by Crescenze [20]. The raw material was found to have a crystallinity of 55.88 $( \pm 1.20) \%$. All samples were tested in triplicate and mean values calculated.

\subsubsection{Tensile testing}

To improve the grip of the samples' during tensile testing, the thinnest ends of the samples were individually fixed to pieces of cardboard using slow-setting adhesive (Araldite Precision, Bostik Ltd). Sample width was measured (Table 2) using a micrometer before being gripped within the tensile tester clamps of the Instron (2211). Samples were tested to failure with a $5 \mathrm{~N}$ load cell and $5 \mathrm{~mm} \mathrm{~min}^{-1}$ crosshead speed. The modulus was determined by comparing two stress-strain points in the initial linear region of the curve. The ultimate tensile strength (UTS) was taken as the highest stress point reached on the curve. Six replicates of each sample type were tested per time point. Mechanical testing was not performed on solvent cast films.

\subsubsection{High performance liquid chromatography (HPLC)}

The PBS surrounding the samples was transferred to vials within the HPLC autosampler. $100 \mu \mathrm{l}$ of sample was injected per run with one injection per sample. All sample types were tested in triplicate. A reverse phase column, with nucleosil packing material with pore size $100 \AA$, particle size $5 \mu \mathrm{m}$, column length $250 \mathrm{~mm}$ and diameter $4.6 \mathrm{~mm}$ (Macherey Nagel), was used. A 0.05 M standard of Capronic acid (Sigma Aldrich) in 20\% acetonitrile and $80 \%$ water was also analysed by HPLC and the chromatograph compared to those produced from the samples. Data acquisition and analysis was determined with Chromeleon 6.60 software (Dionex).

\subsubsection{Electrospray-mass spectrometry (ES-MS)}

By-products, detected by HPLC from a single sample of PBS taken from a three-month degraded sample, were identified by electrospray-mass spectrometry (ES-MS). Prior to sample detection, the ESMS - a Micromass Quattro II triple quadrupole mass spectrometer with a standard electrospray interface (Manchester, UK) - was calibrated with Cesium Iodide and Rubidium Iodide (BDH Chemicals) in deionised water (50:50 mix). Following this, $0.1 \mathrm{mg}$ of PBS from the surrounding sample was added to $1 \mathrm{ml}$ deionised water. $10 \mu \mathrm{l}$ of this solution was injected into the ES-MS with a constant stream of Methanol (Sigma Aldrich), flowing at $0.5 \mathrm{ml} \mathrm{min}^{-1}$. As the sample entered the instrument, the water was removed by a stream of Nitrogen (in house), flowing at $250 \mathrm{~L} \mathrm{~h}^{-1}$, in the presence of a high potential. Only the sample ions remained, which were then passed through the quadrupole to separate them by mass. The high potential was varied to detect both positive and negative ions. Peaks were identified with software Mass Spec Solver II (Manchester University).

\subsubsection{Scanning electron microscopy (SEM)}

Morphological assessment of all samples was determined using a scanning electron microscope (SEM) (Topcon SM300). Samples were gold sputter-coated for 2 minutes to ensure an even coverage. Samples were then visualized in the SEM using an $8 \mathrm{~mm}$ working distance and $5 \mathrm{keV}$ accelerated electron beam. All samples were tested in duplicate.

\subsection{Statistical analysis}

Acquired data was statistically analysed using Graphpad Prism version 3.03 software (Graphpad). Normal distribution of data was

Table 2

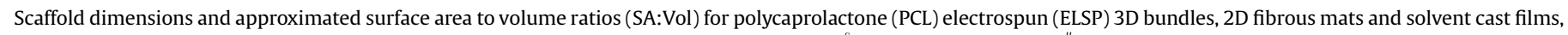
where $\emptyset$ represents the diameter of the bundle, and the shapes of the scaffolds assumed as ${ }^{\S}$ rectangular prism and ${ }^{\#}$ closed cylinder.

\begin{tabular}{|c|c|c|c|c|c|c|}
\hline Scaffold & $\begin{array}{l}\text { Length } \\
\text { (1) }(\mathrm{mm})\end{array}$ & $\begin{array}{l}\text { Width }(\mathrm{w}) \\
(\mathrm{mm})\end{array}$ & $\begin{array}{l}\text { Depth }(\mathrm{d}) \\
(\mathrm{mm})\end{array}$ & $\begin{array}{l}\text { Surface Area } \\
(\mathrm{SA})\left(\mathrm{mm}^{2}\right)\end{array}$ & $\begin{array}{l}\text { Volume } \\
\left(\mathrm{mm}^{3}\right)\end{array}$ & $\begin{array}{l}\text { SA:Vol } \\
\left(\mathrm{mm}^{-1}\right)\end{array}$ \\
\hline ELSP 3D Bundle & 30.00 & $0.35(\varnothing)$ & - & 33.18 & 2.89 & 11.48 \\
\hline ELSP 2D Fibrous Mat ${ }^{\S}$ & 30.00 & 3.00 & 0.16 & 190.56 & 14.40 & 13.23 \\
\hline Solvent Cast Film ${ }^{\S}$ & 30.00 & 3.00 & 0.21 & 193.86 & 18.90 & 10.26 \\
\hline
\end{tabular}


Table 3

Changes in molecular weight $\left(\mathrm{M}_{w}\right)$ and molecular number $\left(\mathrm{M}_{n}\right)$ for polycaprolactone (PCL) electrospun (ELSP) 3D bundles, 2D fibrous mats and solvent cast films for a 90 day degradation study. Data shown for time zero and 90 day degradation only, represented as mean ( \pm standard deviation) ( $n=3$ per sample type). Compared with unpaired $t$-test $(p<0.05)$; ns - not significantly different.

\begin{tabular}{|c|c|c|c|c|c|c|}
\hline & \multicolumn{2}{|c|}{ ELSP 3D Bundle } & \multicolumn{2}{|c|}{$\begin{array}{l}\text { ELSP 2D Fibrous } \\
\text { Mat }\end{array}$} & \multicolumn{2}{|c|}{ Solvent Cast Film } \\
\hline & 0 day & 90 day & 0 day & 90 day & 0 day & 90 day \\
\hline $\mathrm{M}_{w} \mathrm{gmol}^{-1}$ & $\begin{array}{l}174,900 \\
( \pm 8861)\end{array}$ & $\begin{array}{l}141,871 \\
( \pm 3602)\end{array}$ & $\begin{array}{l}180,180 \\
( \pm 4903)\end{array}$ & $\begin{array}{l}148,789 \\
( \pm 4685)\end{array}$ & $\begin{array}{l}142,025 \\
( \pm 1919)\end{array}$ & $\begin{array}{l}123,178 \\
( \pm 991)\end{array}$ \\
\hline$P$ Value & $p<0.05$ & & $p<0.05$ & & $p<0.05$ & \\
\hline $\mathrm{M}_{n} \mathrm{gmol}^{-1}$ & $\begin{array}{l}99,007 \\
( \pm 1737)\end{array}$ & $\begin{array}{l}92,890 \\
( \pm 3815)\end{array}$ & $\begin{array}{l}105,775 \\
( \pm 4972)\end{array}$ & $\begin{array}{l}99,863 \\
( \pm 6419)\end{array}$ & $\begin{array}{l}83,116 \\
( \pm 1519)\end{array}$ & $\begin{array}{l}78,934 \\
( \pm 1605)\end{array}$ \\
\hline$P$ Value & $p<0.05$ & & ns & & $p<0.05$ & \\
\hline
\end{tabular}

confirmed and subsequently analysed with one-way ANOVA and Bonferroni post-tests with 95\% confidence level.

\section{Results}

\subsection{Surface area to volume ratio}

To understand the SA:Vol of the three scaffolds investigated, approximate ratios for each were determined (Table 2); both solvent cast films and 2D fibrous mats were assumed to be rectangular prisms, and 3D bundles treated as a closed cylinder. The results demonstrated highest SA:Vol in the following order; 2D fibrous mats $>3 \mathrm{D}$ bundles $>$ solvent cast films.

\subsection{Molecular mass distribution of PCL samples}

Assessment of molecular mass highlighted an increase in number average molecular mass when compared to the manufac-

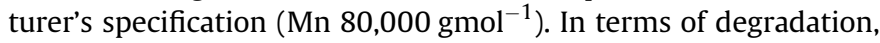
the results highlight a significant decrease in molecular mass distributions for all sample types over the 90 day study (Table 3). Greatest reduction in chain lengths $\left(\mathrm{M}_{n}\right)$, and hence degradation, was observed for 3D bundles (6.2\%), followed by 2D fibrous mats (5.6\%) and then solvent cast films (5.0\%). Similarly, 3D bundles

\section{Table 4}

Change in crystallinity for polycaprolactone (PCL) electrospun (ELSP) 3D bundles, ELSP 2D fibrous mats and solvent cast films over a 90 day degradation period a $37^{\circ} \mathrm{C}$. Data shown as mean ( \pm standard deviation) ( $n=3$ per sample type). Crystallinity of each time point was compared to time zero using one-way ANOVA and Bonferroni post-tests $(p<0.05)$; ns - not significant.

\begin{tabular}{cllll}
\hline & Time-point & \multicolumn{2}{l}{ PCL degradation sample type } & \\
\cline { 3 - 5 } & & ELSP 3D bundle & $\begin{array}{l}\text { ELSP 2D } \\
\text { fibrous mat }\end{array}$ & $\begin{array}{l}\text { Solvent } \\
\text { cast film }\end{array}$ \\
\hline Crystallinity & 0 day & $44.62( \pm 7.58)$ & $53.02( \pm 1.98)$ & $69.53( \pm 1.15)$ \\
$\chi_{c}(\%)$ & 14 day & $52.48( \pm 7.60)$ & $54.11( \pm 0.48)$ & $79.87( \pm 3.76)$ \\
& $P$ Value & ns & ns & $p<0.05$ \\
& 30 day & $61.37( \pm 7.87)$ & $68.31( \pm 1.74)$ & $76.25( \pm 1.48)$ \\
& $P$ Value & $p<0.05$ & $p<0.05$ & $p<0.05$ \\
60 day & $62.85( \pm 1.96)$ & $70.89( \pm 4.09)$ & $79.72( \pm 2.77)$ \\
& $P$ Value & $p<0.05$ & $p<0.05$ & $p<0.05$ \\
90 day & $59.08( \pm 4.25)$ & $58.73( \pm 2.17)$ & $75.88( \pm 0.72)$ \\
$P$ Value & $p<0.05$ & ns & $p<0.05$ \\
\hline
\end{tabular}

experienced greatest loss in molecular weight (18.9\%), compared to 2D fibrous mats (17.4\%) and solvent cast films (13.3\%).

\subsection{Thermal properties and crystallinity of PCL samples}

Comparison of processed samples to the raw material $(55.88 \pm 1.20 \%)$ resulted in a significant change in percentage crystallinity with no obvious trend. However, analysis of the first heat cycle for all samples during the study demonstrated similar trends in melting temperature and enthalpy of fusion (Fig. 2). The melting temperature increased over time and the enthalpy of fusion resulted in an overall increase after 90 days. This increasing trend was similarly observed in sample crystallinity (Table 4). For $3 \mathrm{D}$ bundles and 2D fibrous mats crystallinity peaked at 60 days and after 30 days for solvent cast films.

\subsection{Tensile properties of electrospun PCL samples}

\subsubsection{Modulus}

The modulus followed a similar trend for both electrospun scaffolds with the mean value steadily increasing after 60 days storage in PBS at $37^{\circ} \mathrm{C}$. Stiffness increased by $70 \%$ for $3 \mathrm{D}$ bundles
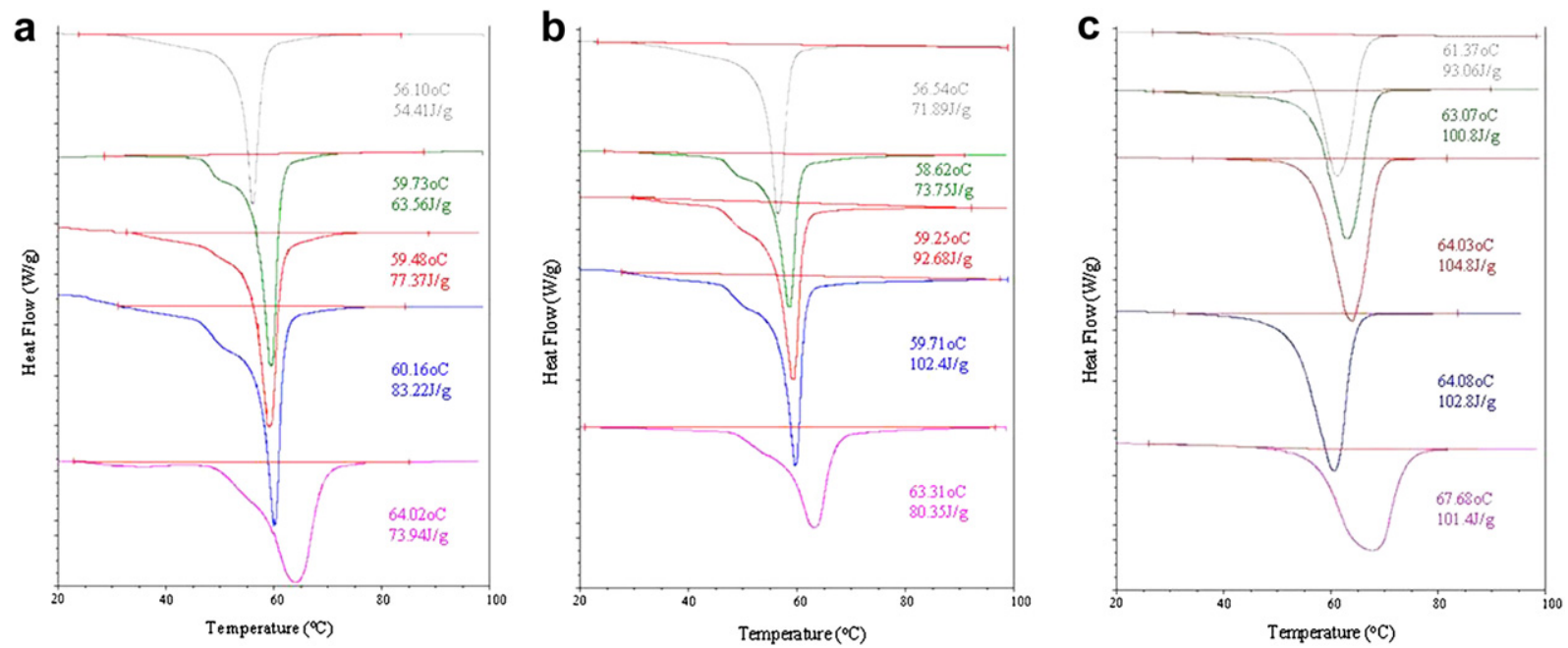

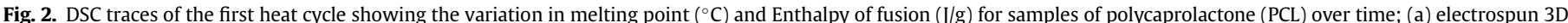

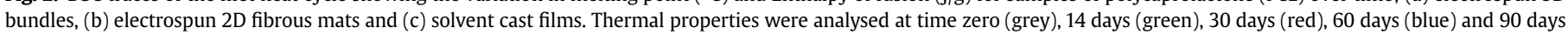
(pink) (For interpretation of the references to color in this figure legend, the reader is referred to the web version of this article.). 


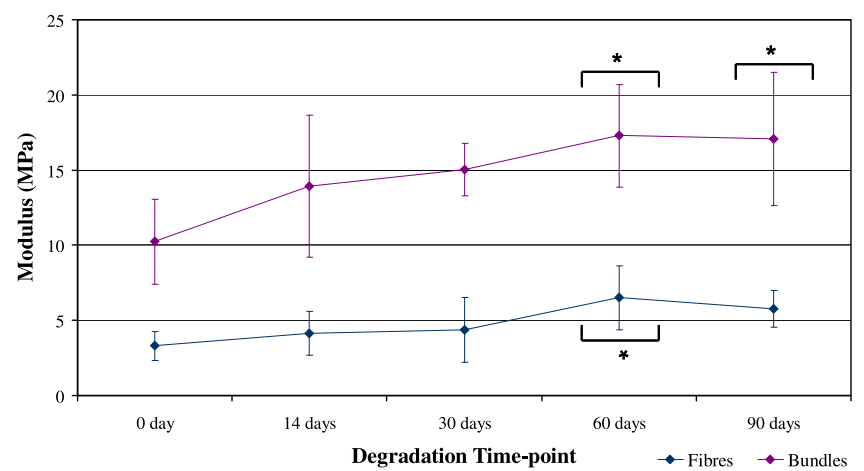

Fig. 3. Modulus obtained for polycaprolactone electrospun 2D fibrous mats (blue) and 3D bundles (purple) following storage at $37^{\circ} \mathrm{C}$ for a total of 90 days. Data shown as mean \pm standard deviation ( $n=6$ per sample type). Modulus compared to time zero using one-way ANOVA and Bonferroni post-tests $\left({ }^{*} p<0.05\right)$.

and $98 \%$ for 2D fibrous mats (when compared to their initial starting values), before decreasing slightly after 90 days (Fig. 3).

\subsubsection{Tensile strength}

The ultimate tensile strength (UTS) of the electrospun scaffolds followed a similar trend to the modulus (Fig. 4). The 2D fibrous mats demonstrated a significant increase in UTS after one month's storage at $37^{\circ} \mathrm{C}$, continuing up to two months. A slight decrease occurred at 90 days; however, the UTS remained significantly higher than time zero - almost doubling in strength. The 3D bundles displayed a steady increase in mean UTS, with values at 60 days and 90 days storage being considerably stronger compared to the initial time-point, with increases of approximately $60 \%$ for both time-points.

\subsection{Analysis of PCL degradation products}

Degradation by-products were identified for all sample types stored in PBS and over the time period investigated the time taken to elute these by-products decreased (Fig. 5(a)), and the level of byproduct peak area was found to increase (Fig. 5(b)). No significant difference between the three scaffold types was observed.

\subsubsection{Identification of $P C L$ degradation products}

Analysis of the surrounding PBS from a 90 day sample was performed by ES-MS. A range of compounds (or oligomers) with

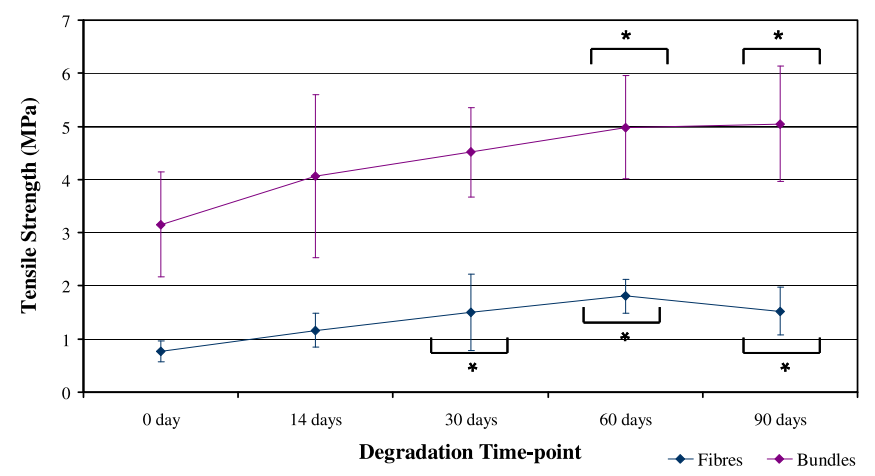

Fig. 4. Tensile strength obtained for polycaprolactone electrospun $2 \mathrm{D}$ fibrous mats (blue) and 3D bundles (purple) following storage at $37^{\circ} \mathrm{C}$ for a total of 90 days. Data shown as mean \pm standard deviation ( $n=6$ per sample type). Tensile strength compared to time zero using one-way ANOVA and Bonferroni post-tests ( ${ }^{*} p<0.05$ ). varying molecular mass were detected; identification of these compounds were found to be of the general capronic acid form (Table 5).

\subsection{Surface analysis of PCL samples}

SEM analysis highlighted no distinct modification in material morphology for all three scaffolds investigated over the 90 day degradation period (Fig. 6).

\section{Discussion}

\subsection{Surface area to volume ratio}

The surface area to volume ratio (SA:Vol) is an important aspect with regards to diffusion pathways. The shapes - rectangular prism and cylinder - assume the surfaces' to be smooth, though this is not the case for the three scaffolds in this study. The surface roughness of the solvent cast film and individual fibres within the electrospun scaffolds have not been taken into account. Hence, the calculated values will be far lower than their true SA:Vol; although they are likely to follow the same trend, being; 2D fibrous mats $>3 \mathrm{D}$ bundles $>$ solvent cast films.

\subsection{Molecular mass distribution of PCL samples}

Molecular mass of all scaffolds was found to increase from the initial Mn $\left(80,000 \mathrm{gmol}^{-1}\right)$. The initial molecular mass for the three different scaffolds was varied, despite scaffolds being fabricated from a single polymer/solvent solution. Reasons for this may be due to the processing technique, i.e. solvent casting and electrospinning methods. When the polymer pellets are dissolved in solvent, it may be possible for the now mobile polymer chains to bond to other free chains, subsequently increasing the chain number. However, this phenomenon is outside the scope of this paper and will not be discussed in any further detail.

Although the greatest molecular mass loss was observed for 3D fibrous bundles, the results on a whole support the hypothesis of Bölgen [10], where samples of higher SA: $\mathrm{Vol}$ are expected to degrade faster due to greater water penetration into their structures. It is possible that 3D fibrous bundles may have experienced greatest loss compared to samples of largest SA:Vol, i.e. 2D fibrous mats, due to released acid becoming localised within the bundle structure causing an autocatalytic reaction. (Note; SA:Vol values are approximate.) Whilst scaffolds of higher SA:Vol possess shorter diffusion pathways, as proposed by Kim [13], the fact the surrounding solutions were not changed during the course of the investigation may have promoted autocatalysis, which would have increased the rate of chain scission.

\subsection{Thermal properties and crystallinity of PCL samples}

Similar to the material's molecular weight, the effects of material processing appear to affect the percentage crystallinity and reasons for this fall outside the aims of this paper.

The thermal changes observed are indicative of hydrolytic attack, sustained by the material bulk, as a consequence of water molecules penetrating the scaffold and targeting ester groups within its amorphous regions [21]. Rearrangement of molecular chains due to chain cleavage creates a more ordered structure, which consequently increases the level of crystallinity [22]. The level of crystal formation is, however, limited and once this had been reached, degradation of the oligomers and breakdown of the crystals can be expected. 

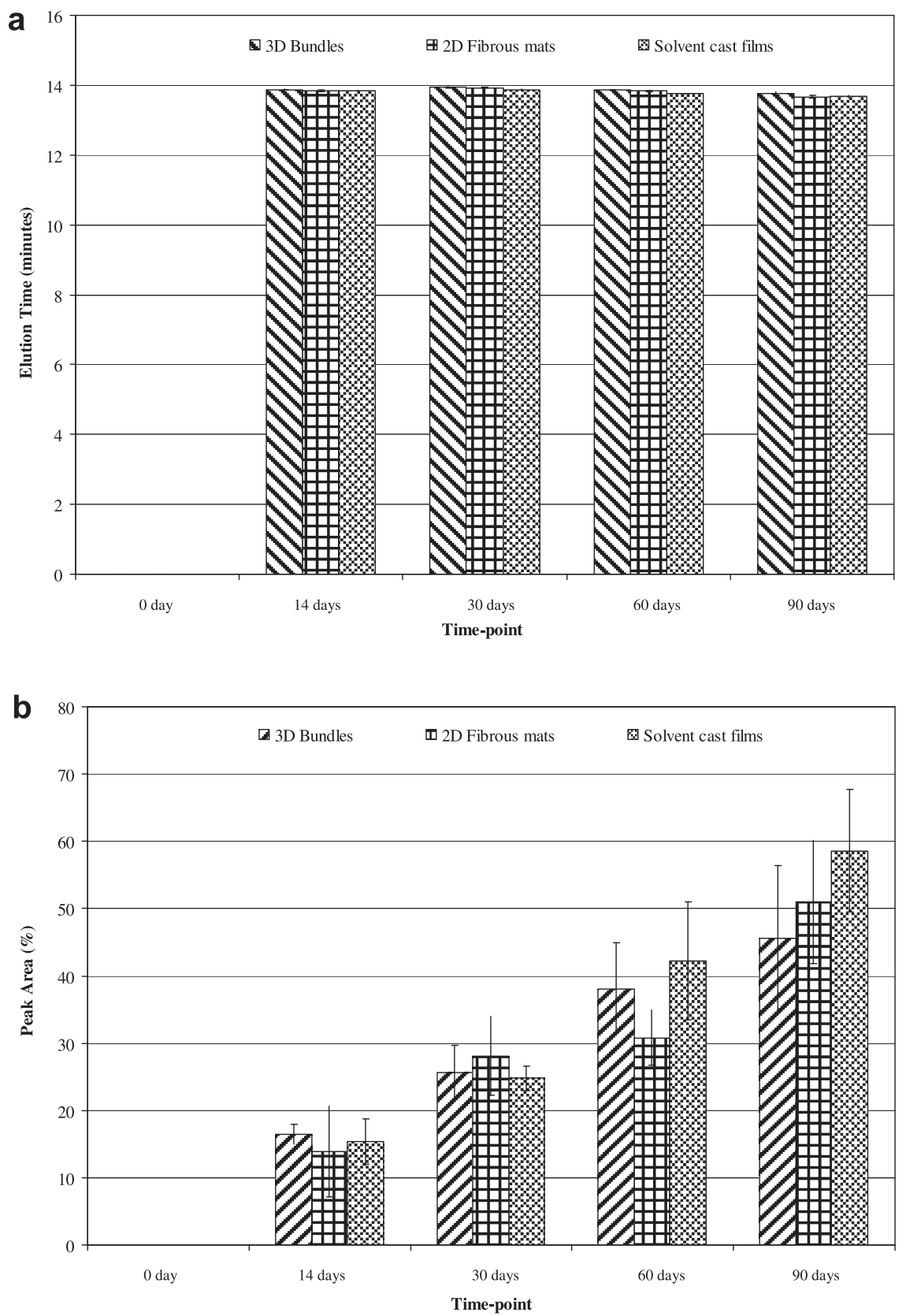

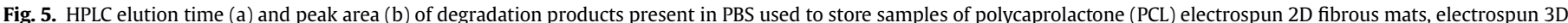

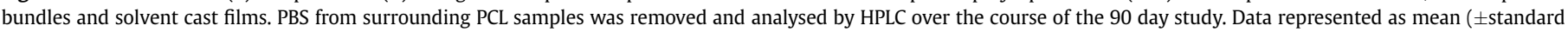
deviation) ( $n=3$ per sample type)

\subsection{Tensile properties of electrospun PCL samples}

\subsubsection{Modulus}

Over the course of the study, the modulus was found to increase for both types of electrospun scaffold. This can be attributed to the crystallinity of the samples', which was found to increase in both sample types over time. A more crystalline structure tends to possess an ordered arrangement; consequently allowing the material to withstand greater loads.

\subsubsection{Tensile strength}

The tensile strength of the electrospun scaffolds followed a similar trend to the modulus, whereby a gradual increase in strength was initially observed with time, followed by a slight decrease (for 2D fibrous mats) after 90 days storage in PBS. This increase in tensile strength for both sample types may again be attributed to their raised levels of crystallinity; thus enabling greater loads to be withstood by the material.

\subsection{Analysis of PCL degradation products}

Degradation by-products within the surrounding PBS solution were detected for all sample types. The time for these by-products to be eluted reduced over the course of the degradation study, which suggests they were becoming more hydrophobic and hence more likely to consist of chains of longer lengths. The increase in by-product peak area for the three sample types is further indicative of bulk degradation, and may have been accelerated by 
Table 5

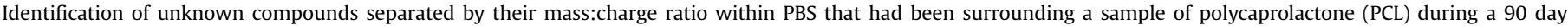
degradation study at $37^{\circ} \mathrm{C}$ as detected by electrospray-MS.

\begin{tabular}{|c|c|c|c|}
\hline $\begin{array}{l}\text { Mass spectrum } \\
\text { Peak Number }\end{array}$ & $\begin{array}{l}\text { Possible Mass } \\
\text { spectrum Match }\end{array}$ & Possible Molecular Identity & $\begin{array}{l}\text { Possible Molecular } \\
\text { Structure }\end{array}$ \\
\hline 139 & 137 & 1 Lactone $1 \mathrm{Na}^{+}$ & Cyclic \\
\hline 269 & 268 & 1 Capronic acid $1 \mathrm{PO}_{4}^{2-} 1 \mathrm{Na}^{+} 1 \mathrm{H}_{2} \mathrm{O}$ & Linear \\
\hline 315 & 317 & 1 Lactone 1 Capronic acid $1 \mathrm{~K}^{+} 1 \mathrm{MeOH}$ & Linear \\
\hline 327 & 324 & 2 Lactone $1 \mathrm{PO}_{4}^{2-} 1 \mathrm{H}^{+}$ & Cyclic \\
\hline 373 & 375 & 3 Lactone $1 \mathrm{MeOH} 1 \mathrm{H}^{+}$ & Cyclic \\
\hline 384 & 382 & 1 Lactone 1 Capronic acid $1 \mathrm{PO}_{4}^{2-} 1 \mathrm{Na}^{+} 1 \mathrm{H}_{2} \mathrm{O}$ & Linear \\
\hline 431 & 431 & 2 Lactone 1 Capronic acid $1 \mathrm{~K}^{+} 1 \mathrm{MeOH}$ & Linear \\
\hline 491 & 489 & 4 Lactone $1 \mathrm{MeOH} 1 \mathrm{H}^{+}$ & Cyclic \\
\hline 491 & 493 & 3 Lactone 1 Capronic acid $1 \mathrm{H}^{+} 1 \mathrm{H}_{2} \mathrm{O}$ & Linear \\
\hline 503 & 505 & 3 Lactone 1 Capronic acid $1 \mathrm{MeOH} 1-$ ve ion & Linear \\
\hline 525 & 527 & 4 Lactone $1 \mathrm{~K}^{+} 1 \mathrm{MeOH}$ & Cyclic \\
\hline 609 & 607 & 4 Lactone 1 Capronic acid $1 \mathrm{H}^{+} 1 \mathrm{H}_{2} \mathrm{O}$ & Linear \\
\hline 609 & 609 & 5 Lactone $1 \mathrm{~K}^{+}$ & Cyclic \\
\hline 609 & 611 & 4 Lactone 1 Capronic acid $1 \mathrm{Na}^{+}$ & Linear \\
\hline 609 & 611 & 5 Lactone $1 \mathrm{Na}^{+} 1 \mathrm{H}_{2} \mathrm{O}$ & Cyclic \\
\hline 796 & 797 & 7 Lactone $1-$ ve ion & Cyclic \\
\hline 796 & 798 & 5 Lactone 1 Capronic acid $1 \mathrm{PO}_{4}^{2-} 1 \mathrm{H}^{+}$ & Linear \\
\hline 796 & 798 & 6 Lactone $1 \mathrm{PO}_{4}^{2-} 1 \mathrm{H}^{+} 1 \mathrm{H}_{2} \mathrm{O}$ & Cyclic \\
\hline
\end{tabular}

autocatalysis occurring secondary to the accumulation of acidic breakdown products.

\subsubsection{Identification of PCL degradation products}

Review of related literature and the fact no enzymes were present within the PBS solution; suggest that the PCL samples underwent partial bulk degradation over the course of the 90 day study. The varied lengths identified by ES-MS also suggest random chain scission occurred within the material bulk; although this was not confirmed by an increase in the polydispersity indices as determined by GPC (data not shown), and may instead be attributed to short capronic acid chains reacting with each other in solution to create either longer chains or cyclic lactones.

\subsection{Surface analysis of PCL samples}

The electron micrographs demonstrated a lack of surface change to the samples. This suggests bioerosion had not occurred and implies the alterations observed in material properties over the 90 day study were caused by hydrolytic attack occurring within the material bulk.
ELSP 3D Fibrous Bundles

0 day

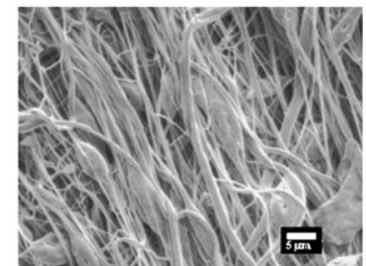

30 days

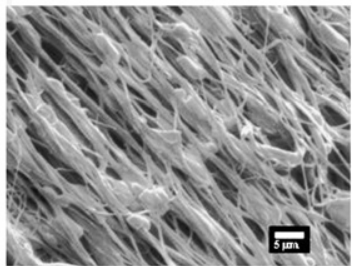

90 days

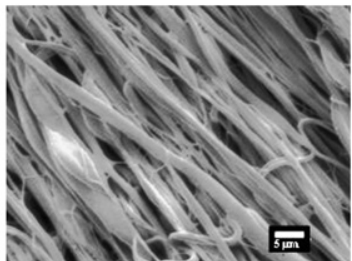

ELSP 2D Fibrous Mats
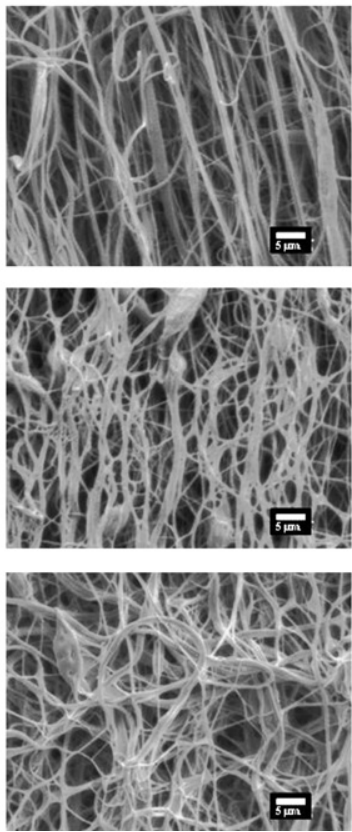

Solvent Cast Films
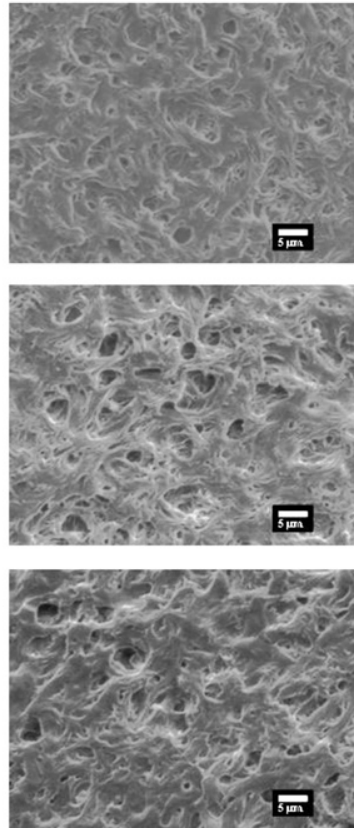

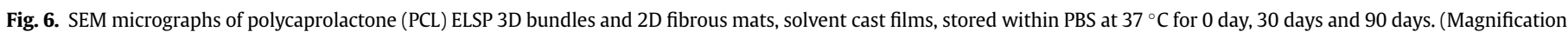
$\times 2000$, bar $5 \mu \mathrm{m}$ ). 


\section{Conclusions}

During the 90 day period investigated, PCL samples demonstrated increased crystallinity, suggesting hydrolytic attack had occurred within the amorphous regions of the polymer, and caused the rearrangement of chains to create a more ordered structure. This was supported by increases in material stiffness and strength typical of higher ordered materials. Changes in the molecular mass distributions over time were greatest for electrospun 3D bundles, followed by electrospun 2D mats and finally solvent cast films. These results support the effects of SA:Vol on diffusion pathways; however, 3D bundles did not have the largest SA:Vol despite undergoing the greatest loss in molecular mass. This may be attributed to 3D bundles having more amorphous regions; thus providing a larger area for hydrolytic attack compared to the 2D fibrous mats. Molecular mass loss may have been further catalysed by the build up of acid within the solution as this remained unchanged throughout the study. Analysis of the surrounding PBS confirmed the presence of short chains (or oligomers) of varying lengths, all with capronic acid end groups (carboxylic acid and alcohol functional groups). The quantity of degradation products released increased over the time period investigated, as determined by HPLC analysis. There were no obvious visual changes in the materials' surface; this is most probably due to the lack of enzymes in the PBS solution used for this study. In conclusion, the degradation of PCL scaffolds, stored in PBS at $37^{\circ} \mathrm{C}$, demonstrated hydrolysis of the material bulk, which was identified by the presence of capronic acid oligomers in the surrounding solution. For the three PCL scaffolds investigated, 3D electrospun bundles demonstrated greatest molecular mass loss, followed by 2D electrospun mats and finally solvent cast films.

\section{Acknowledgements}

This work was funded by the Engineering and Physical Sciences Research Council (EPSRC). The authors would like to thank Dr. Andrew Smith for his assistance with the HPLC and Dr. Paul Christian for use of the ES-MS.

\section{References}

[1] Hutmacher DW. Scaffolds in tissue engineering bone and cartilage. Biomaterials 2000;21(24):2529-43.

[2] Vert M, Li SM, Spenlehauer G, Guerin P. Bioresorbability and biocompatibility of aliphatic polyesters. J Mater Sci Mater Med 1992;3(6):432-46.
[3] Tsuji H, Ono T, Saeki T, Daimon H, Fujie K. Hydrolytic degradation of poly ([epsilon]-caprolactone) in the melt. Polym Degrad Stab 2005;89(2):336-43.

[4] Sun H, Mei L, Song C, Cui X, Wang P. The in vivo degradation, absorption and excretion of PCL-based implant. Biomaterials 2006;27(9):1735-40.

[5] Lam CXF, Savalani AMM, Teoh AS-H, Hutmacher DW. Dynamics of in vitro polymer degradation of polycaprolactone-based scaffolds: accelerated versus simulated physiological conditions. Biomed Mater; 2008:3.

[6] Pitt CG, Hendren RW, SchindIer A, Woodward SC. The enzymatic surface erosion of aliphatic polyesters. J Control Rel 1984;1(3).

[7] Pitt CG, Chasalow FI, Hibionada YM, Klimas DM, Schindler A. Aliphatic polyesters I: the degradation of poly(e-caprolactone) in vivo. J Appl Polym Sci 1981;26:3779-87.

[8] Cook WJ, Cameron JA, Bell JP, Huang SJ. Scanning electron microscopic visualization of biodegradation of polycaprolactones by fungi. J Polym Sci Polym Lett Ed 1981;19:159-65.

[9] Yuan X, Mak AFT, Yao K. Surface degradation of poly(l-lactic acid) fibres in a concentrated alkaline solution. Polym Degrad Stab 2003;79(1):45-52.

[10] Bölgen N, Menceloglu YZ, Acatay K, Vargel I, Piskin E. In vitro and in vivo degradation of non-woven materials made of poly( $\epsilon$-caprolactone) nanofibers prepared by electrospinning under different conditions. J Biomater Sci Polymer Edn 2005;16(12):1537-55.

[11] Holland SJ, Tighe BJ, Gould PL. Polymers for biodegradable medical devices. 1. The potential of polyesters as controlled macromolecular release systems. J Control Rel 1986;4:155-80.

[12] Göpferich A. Mechanisms of polymer degradation and erosion. Biomaterials 1996;17(2):103-14.

[13] Kim K, Yu M, Zong X, Chiu J, Fang D, Seo Y-S, et al. Control of degradation rate and hydrophilicity in electrospun non-woven poly(L-lactide) nanofiber scaffolds for biomedical applications. Biomaterials 2003;24(27):4977-85.

[14] Chen VJ, Ma PX. The effect of surface area on the degradation rate of nanofibrous poly(L-lactic acid) foams. Biomaterials 2006;27(20):3708-15.

[15] Athanasiou KA, Schmitz JP, Agrawal CM. The effects of porosity on in vitro degradation of polylactic acid; polyglycolic acid implants used in repair of articular cartilage. Tissue Eng 1998;4(1):53-63.

[16] Zeng J, Chen X, Liang Q Xu X, Jing X. Enzymatic degradation of Poly(L-lactide) and Poly(e-caprolactone) electrospun fibers. Macromol Biosci 2004;4 (12):1118-25.

[17] Mochizuki M, Hirami M. Structural effects on the biodegradation of aliphatic polyesters. Polym Advan Technol 1997;8(4):203-9.

[18] Ito Y, Hasuda H, Kamitakahara M, Ohtsuki C, Tanihara M, Kang I-K, et al. A composite of hydroxyapatite with electrospun biodegradable nanofibers as a tissue engineering material. J Biosci Bioeng 2005;100(1):43-9.

[19] Gan Z, Liang Q Zhang J, Jing X. Enzymatic degradation of poly(e-caprolactone) film in phosphate buffer solution containing lipases. Polym Degrad Stab 1997;56:209-13.

[20] Crescenze V, Manzini G, Calzolari G, Borri C. Thermodynamics of fusion of poly $\beta$-propiolactone and poly e-caprolactone. Comparative analysis of the melting of aliphatic polylactone and polyester chains. Eur Polym J 1972;8:449-63.

[21] Tsuji H, Mizuno A, Ikada Y. Properties and morphology of poly(L-lactide). III. Effects of initial crystallinity on long-term in vitro hydrolysis of high molecular weight poly(L-lactide) film in phosphate-buffered solution. J Appl Polym Sci 2000;77(7):1452-64.

[22] Kister G, Cassanas G, Bergounhon M, Hoarau D, Vert M. Structural characterization and hydrolytic degradation of solid copolymers of, lactide-co-[e]caprolactone by Raman spectroscopy. Polymer 2000;41(3):925-32.

[23] Shih C. Chain-end scission in acid catalyzed hydrolysis of poly (l-lactide) in solution. J Control Release 1995;34(1):9-15 